\title{
L'utilizzo del test di screening nutrizionale Malnutrition Inflammation Score (MIS) in dialisi
}

\author{
Cristina Borgio ${ }^{1}$, Giacomo Forneris ${ }^{2}$, Andrea Pezzana ${ }^{3}$, Dario Roccatello ${ }^{4}$ \\ ${ }^{1}$ S.O.S.D. Dietetica e Nutrizione Clinica, Ospedale Giovanni Bosco, Torino \\ ${ }^{2}$ SCUD Nefrologia e Dialisi, Ospedale Giovanni Bosco, Torino \\ ${ }^{3}$ Responsabile SOSD Dietetica e Nutrizione Clinica, Ospedale Giovanni Bosco, Torino \\ ${ }^{4}$ Direttore SCUD Nefrologia e Dialisi, Ospedale Giovanni Bosco, Torino
}

\begin{abstract}
The use of the nutritional screening test Malnutrition Inflammation Score (MIS) in dialysis
Protein-energy malnutrition is one of the most common complications in chronic renal failure patients who are treated with hemodialysis, especially in the elderly and in patients who have a long dialysis history.

Protein-energy malnutrition is associated with higher rates of mortality and morbidity, and also with a greater risk of cardiovascular death in dialysis patients.

The importance of nutritional status evaluation and treatment is underlined by some guidelines, such as Kidney Disease Outcomes Quality Initiative.

Nutritional screening should be an easy and fast tool that should be undertaken during the first meeting with the patient and when the patient is hospitalized or registered in other institutions.

Scientific literature (see for example Kalantar-Zadeh et al) shows that Malnutrition Inflammation Score (MIS) seems to have a high capability in discriminating malnutrition, inflammatory status and anemia and that it seems to be highly predictive for morbidity (number of hospitalizations) and mortality.

The objectives of these studies were the perspective of the administration of the MIS to a group of patients treated with hemodialysis in the SCUD (Departmental and University Complex Structure) of Dialysis and Nephrology of Saint Giovanni Bosco Hospital in Turin and the evaluation of the nutritional intervention effects. One of the goal of this study was the involvement of registered nurses, under the coordination of the registered dietitian, in order to have an integrated approach to the problem.

In our experience, the monitoring of patients treated with hemodialysis who have been screened to evaluate their nutritional status with an easy and repeatable procedure allows to express some considerations.

If we keep in mind the common shortage of registered dietitians in dialysis units, being able to engage registered nurses trained and sensible to the problem of malnutrition, it represents a useful tool to improve the quality of dialysis patients life. Being aware of the MIS is an added value to the professional knowledge of the registered nurses in dialysis units and even more when a professional relation is created between the registered dietitian and the medical doctor involved. Focusing on malnutrition becomes essential because of the changing of the population in dialysis we are observing and for this reason it is important to have and use low-cost screening procedures.

The experience of the Dietetic and Clinical Nutrition Unit and of the Dialysis Unit is a very positive one, thanks to the sharing of this project that highlights a multidisciplinary and multiprofessional approach.
\end{abstract}

Keywords: Hemodialysis, Malnutrition, Prevention, Therapy, Malnutrition Inflammation Score (MIS)

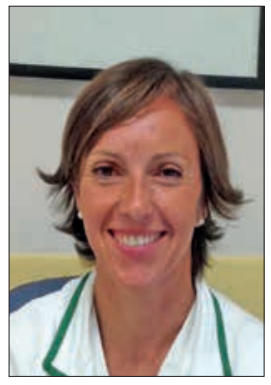

Cristina Borgio

\section{Introduzione}

La malnutrizione proteico-calorica è una delle principali complicanze che possono presentarsi nei pazienti con insufficienza renale cronica sottoposti a trattamento dialitico, con un'incidenza maggiore in quelli anziani e con età dialitica avanzata $(1,2)$.

Come descritto in letteratura, esistono due tipi di malnutrizione proteico-ca-

Accepted: October 12, 2015

Published online: November 5, 2015

Indirizzo per la corrispondenza:

Borgio Cristina

Presidio Ospedaliero San Giovanni Bosco, ASL TO2, Torino

S.o.S.D. Dietetica e Nutrizione Clinica

Piazza del Donatore di Sangue 3, 10154 Torino

cristina.borgio@libero.it lorica: la prima è dovuta a uno scarso intake di nutrienti definito "true malnutrition". In questo caso, non vi sono frequenti condizioni di comorbilità e l'albumina sierica è solitamente normale o leggermente ridotta. Questo tipo di malnutrizione può essere trattato con un apporto proteico-calorico bilanciato.

La seconda è caratterizzata da una riduzione del pool proteico a livello viscerale e somatico causata dal catabolismo legato allo stato infiammatorio. È associata, infatti, a un'infiammazione e a patologie cardiovascolari aterosclerotiche, conosciute come Malnutrition Inflammation Atherosclerosis (MIA syndrome). In questa condizione sono spesso presenti fattori comorbidi e i livelli di albumina sierica sono, in genere, ridotti.

Questo tipo di malnutrizione è difficile da correggere con l'intervento nutrizionale, a meno che non siano diagnosticate e trattate in modo adeguato le condizioni di morbilità presenti e la risposta cronica infiammatoria.

La malnutrizione proteico-calorica è associata a un rischio maggiore di mortalità e morbilità nonché di morte per cause cardiovascolari nei pazienti dializzati. Vari sono i meccanismi 
coinvolti per spiegare il legame tra malnutrizione proteicocalorica e mortalità nell'insufficienza renale cronica, tra cui l'alterazione del tessuto muscolare e adiposo, dell'apparato gastrointestinale e del sistema immunitario ed ematologico come anche l'eccessiva risposta infiammatoria e il possibile sviluppo di patologie cardiovascolari (3).

È noto che, nel $10-30 \%$ dei pazienti in dialisi, la malnutrizione per difetto si associa alla presenza di infiammazione e allo sviluppo di aterosclerosi e costituisce una delle principali cause di elevata mortalità in questi pazienti (circa il 50\% a tre anni dall'inizio del trattamento sostitutivo) (4) e anche di un aumento del tasso di ricoveri ospedalieri (5).

I dati del Registro Italiano di Dialisi e Trapianto indicano che, nella nostra regione, nel 2009, la prevalenza dei pazienti in trattamento sostitutivo è di 3149 , pari a $715 / \mathrm{pmp}$, di cui I'88.5\% in emodialisi $(6,7)$.

È riportata in numerosi studi la stretta associazione tra lo stato nutrizionale, di cui l'albumina sierica è utilizzata come indicatore più importante, e la sopravvivenza del paziente (8).

Sulla base di questi dati è maturato un diffuso consenso sull'impegno necessario per il mantenimento e il recupero di un corretto stato di nutrizionale nei pazienti nefropatici.

L'importanza della valutazione dello stato nutrizionale e del suo adeguato trattamento è messa in evidenza da alcune Linee Guida, tra cui le DOQI (Kidney Disease Outcomes Quality Initiative) (9).

Secondo queste ultime, la valutazione nel paziente in dialisi dovrebbe essere eseguita usando una combinazione di misure valide e complementari piuttosto che una singola determinazione, non disponendo, come è lecito attendersi, di un singolo parametro che fornisca un'indicazione esaustiva dello stato metabolico-nutrizionale.

Nell'ottica di prevenire la malnutrizione, due aspetti sono da considerare prioritari: la necessità di operare uno screening dei pazienti a rischio e, in questi ultimi, la necessità di stabilire il grado di malnutrizione e di valutare l'andamento in risposta alle misure preventive e terapeutiche adottate.

Al momento della presa in carico del paziente, può essere, perciò, indicato l'utilizzo di un test di screening per quantificare e monitorare lo stato di malnutrizione (10) che sia in grado di identificare in modo semplice e veloce e con elevate sensibilità e specificità i pazienti malnutriti, evitando il ricorso ad altri esami più dispendiosi in termini monetari e di tempo (11).

Lo screening nutrizionale dovrebbe essere, quindi, un metodo rapido e semplice, somministrabile a tutti i pazienti all'accettazione in ospedale o in un'altra istituzione (12).

Kalantar-Zadeh et al hanno pubblicato la loro esperienza con il Malnutrition Inflammation Score (MIS), test che comprende 7 componenti del Subjective Global Assessment (SGA) e altri 3 non-SGA: I'indice di massa corporea (BMI), l'albumina sierica e la capacità ferro-legante totale (TIBC) (13).

Negli elementi convenzionali del SGA sono compresi: storia medica, assunzione di cibo, sintomi gastrointestinali, capacità funzionale, comorbilità e durata del trattamento dialitico. L'e- same fisico è costituito da 2 componenti: perdita di grasso sottocutaneo e atrofia muscolare. Ognuna delle 10 componenti del MIS mostra 4 livelli di gravità: da 0 (normale) a 3 (molto grave); la somma di tutte le componenti varia da 0 (normale) a 30 (gravemente malnutrito). Nello studio di riferimento, il punteggio soglia per malnutrizione è stato definito come 6, poiché lo strumento di screening dovrebbe essere in grado di identificare la maggior parte dei pazienti a rischio nutrizionale.

In letteratura, il MIS ha mostrato un'elevata capacità discriminante di malnutrizione, stato infiammatorio e anemia ed è risultato altamente predittivo di morbilità (numero di ospedalizzazioni) e mortalità $(11,14,15)$.

Nella Tabella I sono raffigurate le principali differenze tra il MIS e altri test di screening presenti in letteratura, come MNA-SF, NRS e MUST (16).

Scopo del nostro lavoro è stato rappresentato dalla somministrazione prospettica del MIS a un gruppo di pazienti in trattamento emodialitico presso la SCUD Nefrologia e Dialisi dell'Ospedale Giovanni Bosco di Torino e dalla valutazione degli effetti dell'intervento nutrizionale. Tra gli scopi del lavoro si cita quello di coinvolgere in prima persona il personale infermieristico sotto il coordinamento delle dietiste per un approccio integrato al problema.

\section{Casistica e metodi}

La somministrazione del test è avvenuta da parte degli infermieri, dopo un percorso di formazione sotto la guida del servizio di dietologia che ha illustrato le finalità e le modalità dello strumento di screening MIS.

Il test è stato somministrato nel corso della seduta dialitica e, prima dell' intervista, l'infermiere ha spiegato ai pazienti in dettaglio finalità e metodi dell'indagine. II tempo di somministrazione del test è stato mediamente intorno ai 10 minuti.

La raccolta delle informazioni è avvenuta su un formato cartaceo e, in un secondo momento, è stata elaborata su supporto informatico.

TABELLA I - Variabili relative ai test di screening nutrizionali

\begin{tabular}{lcccc}
\hline Variabili & MIS & MNA-SF & NRS & MUST \\
\hline Perdita di peso o BMI (o entrambi) & $\mathrm{V}$ & $\mathrm{V}$ & $\mathrm{V}$ & $\mathrm{V}$ \\
Assunzione alimentare & $\mathrm{V}$ & $\mathrm{V}$ & & $\mathrm{V}$ \\
Sintomi gastrointestinali & $\mathrm{V}$ & & & \\
Comorbidità & $\mathrm{V}$ & $\mathrm{V}$ & $\mathrm{V}$ & $\mathrm{V}$ \\
Funzionalità fisica & $\mathrm{V}$ & $\mathrm{V}$ & & $\mathrm{V}$ \\
Segni di muscoli o perdita di grasso & & $\mathrm{V}$ & & \\
Problemi psicologici & & $\mathrm{V}$ & & \\
Albumina sierica & & & & \\
Totale capacità ferro-legante & & $\mathrm{V}$ & &
\end{tabular}


II MIS è stato somministrato a 220 pazienti in trattamento emodialitico cronico nel periodo compreso tra il Marzo del 2012 e l'Agosto del 2014, complessivamente 136 uomini e 84 donne con età media pari a $67.7 \pm 14.6$ anni (mediana 70).

Durante questo periodo, sono stati persi all'osservazione 53 pazienti (24.1\%) per decesso (evento verificatosi con maggiore frequenza nella fascia d'età compresa tra gli 80 e gli 89 anni, come atteso $(21 / 53)$.

II progetto ha coinvolto un'equipe multiprofessionale composta da Nefrologi, Dietisti, Dietologi e Infermieri. Un gruppo ristretto di infermieri è stato coinvolto e formato ad hoc all'utilizzo dello strumento da parte del dietista referente della dialisi.

In un gruppo di pazienti (78) non è stato possibile somministrare il test per motivi diversi (barriere linguistiche, condizioni cliniche, cognitive e culturali).

L'analisi statistica è stata eseguita con software STATA 10 e le curve di sopravvivenza sono state calcolate secondo Kaplan-Meier.

\section{Risultati}

Sulla base dello score del MIS, ottenuto alla valutazione iniziale (Marzo 2012), è stato possibile classificare i pazienti in tre gruppi (MIS 1-2-3) in accordo con altre esperienze (17-19).

Nel gruppo a basso rischio di malnutrizione (score 0-5, MIS 1), sono rientrati 65 pazienti (45.8\%), nel gruppo a rischio intermedio (score 6-10, MIS 2) 60 pazienti (42.3\%), mentre 17 pazienti (11.9\%) presentavano uno stato di malnutrizione moderata-grave (score $\geq 11$ MIS 3 ).

I pazienti più anziani anagraficamente e, dal punto di vista dialitico, all'inizio dello studio presentano più elevati livelli di MIS score (Tab. II).

Nel periodo successivo, il test è stato ripetuto in tutti i pazienti presenti al follow-up con cadenza semestrale.

Sulla base dei dati raccolti, è stato possibile eseguire un'analisi al termine del periodo di 2 anni di osservazione sulla popolazione campione.

Nel gruppo MIS 1 (65 pazienti), 40 (61.5\%) sono rimasti stabili, 14 (21.5\%) sono passati nella classe MIS 2 e 2 (3.0\%) alla classe MIS 3, mentre 9 (13.8\%) sono deceduti.

Nel gruppo MIS 2 (60 pazienti), in 21 (35\%) lo score è rimasto stabile, $10(16.6 \%)$ sono passati a uno score migliore di 1, 5 (8.3\%) sono passati a MIS 3 e 24 (40\%) sono deceduti.

Nel gruppo MIS 3 a elevato rischio di malnutrizione, comprendente 17 pazienti, 4 (23.5\%) sono rimasti invariati, 5 (29.4\%)

TABELLA II - Età media per gradi di score MIS

\begin{tabular}{lc}
\hline MIS score & Età media dei pazienti \\
\hline $0-5$ & 65 \\
$6-10$ & 73 \\
$>10$ & 75 \\
\hline
\end{tabular}

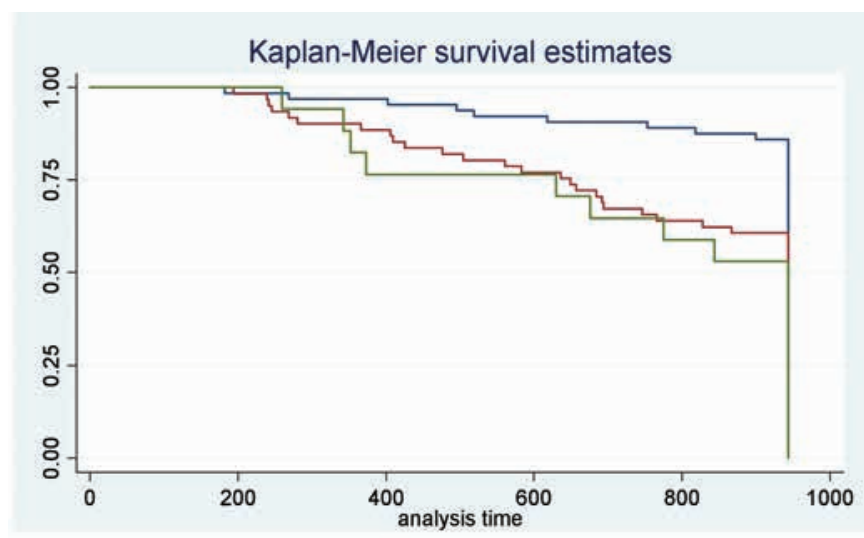

MIS 1: linea blu; MIS 2: linea rossa, MIS 3: linea verde.

Fig. 1 - Sopravvivenza dei pazienti per gradi di MIS score a inizio osservazione.

sono passati a uno score MIS 2 e 8 (47\%) sono deceduti.

I pazienti classificati con un livello di MIS basso (linea blu, Fig. 1) hanno un tasso di sopravvivenza superiore rispetto ai pazienti con MIS medio-grave (linea rossa e linea verde). L'analisi della curva di Kaplan-Meier mostra una differenza significativa tra i pazienti con MIS medio-grave e i pazienti con MIS lieve. In media, i pazienti con un MIS lieve hanno una probabilità di sopravvivenza superiore rispetto ai pazienti con MIS medio-grave di quattro mesi (p 0.0013) (Fig. 1).

Considerando solo i 17 pazienti con MIS $\geq 11$ alla prima valutazione, a distanza di 2 anni 8 pazienti sono deceduti, 4 hanno avuto un MIS invariato e 5 sono passati a un valore di MIS con score 6-10.

Non si notano differenze nella sopravvivenza tra i pazienti che hanno mantenuto un MIS stabile e quelli che hanno registrato un miglioramento.

Si nota, invece, un significativo maggior rischio di morte per i pazienti che hanno registrato un peggioramento nel valore del MIS >2 punti (Fig. 2).

Durante il follow-up sono stati proposti una terapia nutrizionale a tutti i pazienti malnutriti a diversi livelli, un counselling dietistico, supplementi nutrizionali orali o l'avvio di una nutrizione parenterale intradialitica.

Nel 2012 , il $47 \%$ dei pazienti ha rifiutato la terapia nutrizionale, il $35 \%$ ha integrato la dieta con dei supplementi nutrizionali orali e al $18 \%$ è stato proposto un supporto parenterale.

Nel 2013, il 36\% dei pazienti ha rifiutato la terapia nutrizionale, il 59\% ha integrato la dieta con dei supplementi nutrizionali orali e al $5 \%$ è stato proposto un supporto parenterale.

Nel 2014, il 40\% dei pazienti ha rifiutato la terapia nutrizionale, il $56 \%$ ha integrato la dieta con dei supplementi nutrizionali orali e al $4 \%$ è stato proposto un supporto parenterale.

Non sono emerse differente significative di sopravvivenza tra i pazienti che hanno accettato o rifiutato il supporto nutrizionale (Fig. 3).

L'accettazione del supplemento nutrizionale è stato supe- 


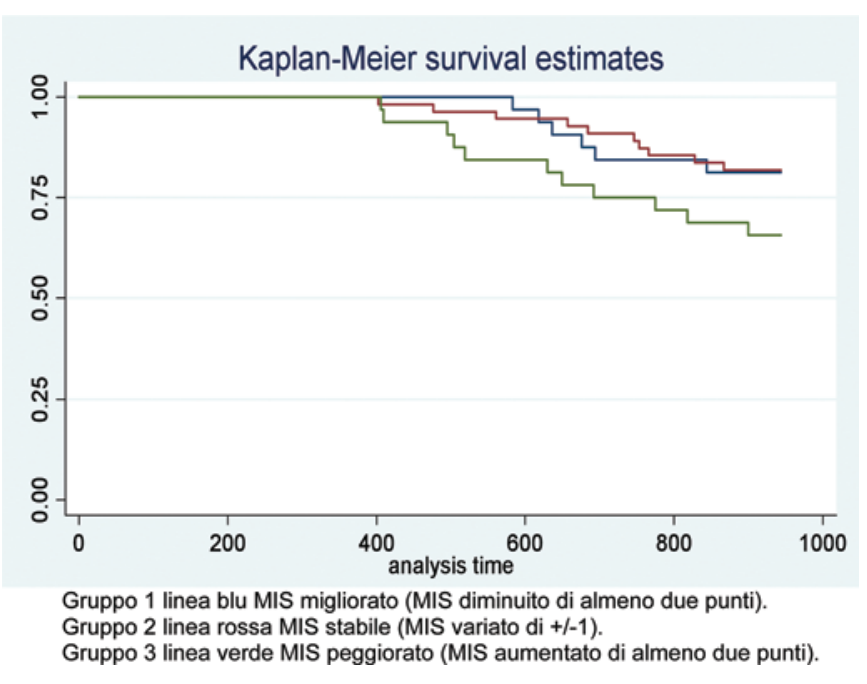

Fig. 2 - Curva di sopravvivenza dei paziente con MIS $\geq 11$ iniziale rispetto alla variazione del punteggio a distanza di 2 anni.

riore nei pazienti con livelli medi di MIS più elevati per ciascuno degli anni di osservazione (2012-2014).

Della popolazione presa in esame 19 pazienti su 220, pari all' $8.6 \%$, sono stati trapiantati, con un'età media dialitica di 3451 giorni.

\section{Discussione}

I pazienti con insufficienza renale cronica, nei vari stadi della malattia, spesso riducono l'intake proteico-calorico per effetto di fattori diversi come l'uremia di per sé con il suo effetto anoressizzante e la restrizione dietetico-proteica nella fase predialitica; inoltre, si verifica una perdita di nutrienti durante la seduta dialitica, a fronte dell'aumento dei fabbisogni nutrizionali.

Come noto, nei pazienti in trattamento dialitico cronico, sono indicati maggiori intake calorici (30-35 Kcal $/ \mathrm{kg} /$ die) e proteici (1.2 g/kg/die) (21-23).

In questo ambito, l'intervento nutrizionale, quando intrapreso tempestivamente, potrebbe contribuire all'incremento dell'assunzione degli alimenti e alla prevenzione o al trattamento della malnutrizione attraverso interventi specifici: counselling dietistico, supplementazione nutrizionale orale e nutrizione artificiale di tipo enterale o parenterale intradialitica.

Secondo quanto proposto dall'International Society of Renal Nutrition and Metabolism (ISRNM), indicatori di un'inadeguata nutrizione sono: punteggio allo screening MIS $\geq 5$, albumina sierica $\leq 3.8 \mathrm{~g} / \mathrm{dL}$ o prealbumina $\leq 28 \mathrm{mg} / \mathrm{dL}$, scarso appetito e alimentazione ridotta, apporto proteico stimato $<1.2 \mathrm{~g} / \mathrm{Kg} /$ die e calorico $<30 \mathrm{Kcal} / \mathrm{kg} /$ die e calo ponderale $>5 \%$ in tre mesi.

Nella nostra esperienza, l'osservazione di una popolazione di emodializzati a cui è stato somministrato in modo prospettico uno strumento di screening nutrizionale semplice e ripetibile, ci permette di esprimere alcune semplici considerazioni.

Come già presente in altri lavori, il MIS si conferma un indi-

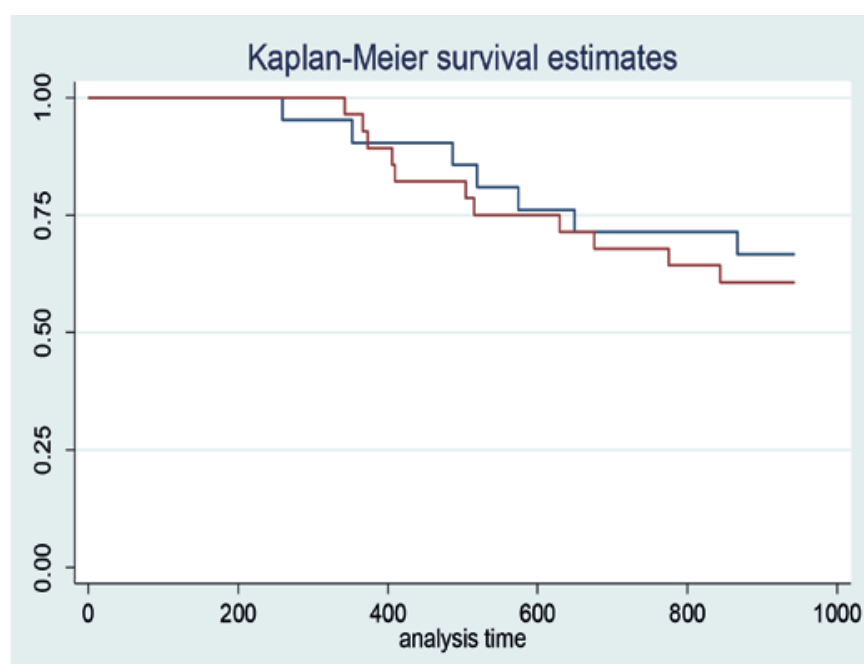

Fig. 3 - Linea rossa: pazienti che hanno accettato il trattamento. Linea blu: pazienti che non hanno accettato il trattamento.

catore di rischio di mortalità del paziente in cui lo stato nutrizionale non è buono, in particolare per punteggi $>6$ e anche per incrementi $>2$ nel corso dell'osservazione. La semplice conferma di questo strumento, che va a integrarsi con altri parametri ematochimici routinari dello stato nutrizionale, ha indubbiamente il vantaggio di mettere a fuoco in modo rapido e semplice i pazienti a rischio, con possibilità di avviare un intervento nutrizionale precoce. È da sottolineare, tuttavia, come, nella nostra esperienza, in molti pazienti il supporto nutrizionale per via orale non sia stato accettato per scarsa palatabilità o per incostanza nei tempi e nelle quantità di consumo, elemento che impedisce un'analisi più puntuale e corposa dell'effetto dell'intervento terapeutico. L'approccio nutrizionale dovrà mirare a individuare le situazioni più precoci di rischio, per proporre il trattamento nutrizionale con lo scopo di impedire il peggioramento e di contenere il MIS score $<6$.

In modo abbastanza atteso, i pazienti con valori di MIS più elevato hanno accettato in misura maggiore l'intervento terapeutico proposto.

Sappiamo che l'intervento nutrizionale parenterale nella malnutrizione grave ha degli effetti clinici dibattuti. La nutrizione parenterale intradialitica (NPID) permette di somministrare calorie e proteine nel corso della dialisi, inducendo un significativo aumento della sintesi delle proteine corporee e una riduzione del catabolismo proteico $(24,25)$, ma il ritmo e il tempo di somministrazione ne limitano certamente l'efficacia nel paziente ambulatoriale.

Al di là dei risultati non precisamente quantificabili, quello che riteniamo importante è il risvolto strategico e organizzativo che l'adozione di uno strumento di screening come il MIS può implicare. Se consideriamo la scarsità delle risorse di personale dietistico a disposizione dei centri dialisi, poter coinvolgere un personale infermieristico motivato e culturalmente attento alla problematica della malnutrizione rappre- 
senta un potenziale strumento di miglioramento della qualità di vita per i pazienti. La conoscenza del MIS costituisce un valore aggiunto al bagaglio professionale dell'infermiere di dialisi, tanto più nel momento in cui si instaurano una relazione e un feedback con la dietista e il medico nefrologo. Porre una maggiore attenzione alla malnutrizione per difetto appare fondamentale con il cambiamento della popolazione dialitica che stiamo osservando e, per questo motivo, è importante avere e utilizzare risorse di screening a basso costo. La partecipazione degli infermieri in questo approccio riduce i tempi di risposta che potremmo avere con il solo intervento del dietista.

L'esperienza del Servizio di Dietetica e Nutrizione Clinica e della Dialisi è da considerare molto positiva grazie alla condivisione di un progetto che avvalora il processo multidisciplinare e multiprofessionale.

Il passo successivo di questo percorso sarà la costituzione di un percorso articolato secondo l'Accademy of Nutrition and Dietetics con l'applicazione del Nutrition Care Process $(26,27)$.

\section{Conclusione}

La prevenzione e il trattamento della malnutrizione sono fondamentali nel paziente emodializzato cronico e si avvalgono dell'utilizzo di interventi nutrizionali a più livelli. Al momento attuale, non esistono trial clinici, prospettici e randomizzati con un numero di pazienti adeguato che permetta conclusioni definitive in merito a specifici trattamenti.

Uno dei macro-obiettivi nella presa in carico del paziente emodializzato deve, quindi, diventare un maggiore impegno nella prevenzione della malnutrizione grave e del suo peggioramento.

II MIS rappresenta uno strumento di valutazione nutrizionale per i pazienti in emodialisi, ma, dal momento che comprende una valutazione soggettiva, richiede un'accurata formazione del personale per garantire risultati coerenti tra diversi esaminatori e tempi diversi.

Secondo la nostra esperienza, il MIS si è dimostrato uno strumento di valutazione nutrizionale completo, pratico, riproducibile e specifico per i pazienti in dialisi e oltremodo rapido e semplice da somministrare da parte del personale infermieristico formato ad hoc.

\section{Disclosures}

Si ringraziano il coordinatore infermieristico e gli infermieri della SCUD Nefrologia e Dialisi, Ospedale Giovanni Bosco, Torino.

\section{Disclosures}

Financial support: No financial support was received for this submission.

Conflict of interest: The authors have no conflict of interest.

\section{Bibliografia}

1. Gama-Axelsson $T$, Heimbürger $O$, Stenvinkel $P$, Bárány $P$, Lindholm B, Qureshi AR. Serum albumin as predictor of nutritional status in patients with ESRD. Clin J Am Soc Nephrol. 2012;7(9):1446-53.

2. Brunori G. Lo stato nutrizionale nel paziente con malattia renale cronica [Nutritional status of patients with chronic kidney disease]. G Ital Nefrol. 2012;29(Suppl.58):S68-71.

3. Cupisti A. Protein-Energy malnutrition in dialysis patients and morbidity/mortality consequence. In: Di lorio B, Heidland A, Ronco C, Onuigbo M, eds: Hemodialysis, when, how, why. Nova Science Publishers, Inc. New York, USA. 2012;341-52.

4. Weiner DE, Tighiouart H, Ladik V, Meyer KB, Zager PG, Johnson DS. Oral intradialytic nutritional supplement use and mortality in hemodialysis patients. Am J Kidney Dis. 2014;63(2):276-85.

5. Ikizler TA, Cano NJ, Franch H. Prevention and treatment of protein energy wasting in chronic kidney disease patients: a consensus statement by the International Society of Renal Nutrition and Metabolism. Kidney Int. 2013;84(6):1096-107.

6. Kadiri Mel M, Nechba RB, Oualim Z. Factors predicting malnutrition in hemodialisys patients. Saudi J Kidney Dis Transpl. 2011;22(4):695-704.

7. Toledo FR, Antunes AA, Vannini FC, et al. Validity of malnutrition scores for predicting mortality in chronic hemodialysis patients. Int Urol Nephrol. 2013;45(6):1747-52.

8. Kalantar-Zadeh K, Ikizler TA. Let Them Eat During Dialysis: An Overlooked Oortunity to Improve Outcomes in Maintenance Hemodialysis Patients. J Ren Nutr. 2013;23(3):157-63.

9. Kopple JD. National kidney foundation K/DOQI clinical practice guidelines for nutrition in chronic renal failure. Am J Kidney Dis. 2001;37(1 Suppl. 2):S66-70.

10. Bossola M, Muscaritoli M, Tazza L, et al. Malnutrition in hemodialysis patients: what therapy? Am J Kidney Dis. 2005;46:371-86.

11. Bossola M, Tazza L, Vulpio $C$, et al. La malnutrizione nel paziente in emodialisi cronica: prevalenza, patogenesi, terapia. [Malnutrition in patients on chronic hemodialysis: prevalence, pathogenesis, and treatment]. G Ital Nefrol. 2009;26(2):201-14.

12. ESPEN Guidelines for Nutrition Screening 2002.

13. Kalantar-Zadeh K, Kopple JD, Block G, Humphreys MH. A malnutrition-inflammation score is correlated with morbidity and mortality in maintenance hemodialysis patients. Am J Kidney Dis. 2001;38:1251-63.

14. Bonanni A, Mannucci I, Verzola D, et al. Protein-energy wasting and mortality in chronic kidney disease. Int J Environ Res Public Health. 2011;8(5):1631-54.

15. Rambod M, Bross R, Zitterkoph J, et al. Association of malnutrition-inflammation score with quality of life and mortality in hemodialisys patients: a 5-year prospective cohort study. Am J Kidney Dis. 2009;53(2):298-309.

16. Kalantar-Zadeh K, Kopple JD, Humphreys MH, Block G. Comparing outcome predictability of markers of malnutritioninflammation complex sydrome in hemodialysis patients. Nephrol Dial Transplant. 2004;19:1507-19.

17. Stevinkel $P$, Heimbürger $O$, Lindholm B, Kaysen GA, Bergström J. Are there two types of malnutrition in chronic renal failure? Evidence for relationship between malnutrition, inflammation and atherosclerosis (MIA syndrome). Nephrol Dial Transplant. 2000;15:953-60.

18. Kalanthar-Zadeh K, Kopple J, Block G, Humphreis M. A Malnutrition-Inflammation Score is correlated with morbidity and mortality in maintenance haemodialysis patients. Am J Kidney Dis. 2001;38(6):1251-63.

19. Casey J, Johnson V, McClelland P. The relationship between 
interdialytic weight gain and patient compliance. A single centred cohort study $(n=21)$. EDTNA ERCA J. 2001;27(4):187-9.

20. Toigo G, Aparicio M, Attman PO, et al. Expert working group report on nutrition in adult patients with renal insufficiency (Part 2 of 2). Clin Nutr. 2000;19(4):281-91.

21. ASPEN Board of Directors and the Clinical Guidelines Task Force. Guidelines for the use of parenteral and enteral nutrition in adult and pediatric patients. JPEN J Parenter Enteral Nutr. 2002;26(Suppl. 1):1SA-138SA.

22. K/DOQI, National Kidney Foundation. Clinical practice guidelines for chronic renal failure. Am J Kidney Dis. 2000;35(Suppl. 2):S1-140.

23. Toigo G, Aparicio M, Attman PO, et al. Expert Working Group report on nutrition in adult patients with renal insufficiency (part 1 of 2). Clin Nutr. 2000;19(3):197-207.

24. Pupim LB, Flakoll PJ, Brouillette JR, Levenhagen DK, Hakim RM, Ikizler TA. Intradialytic parenteral nutrition improves protein and energy homeostasis in chronic hemodialysis patients. J Clin Invest. 2002;110:483-92.

25. Pupim LB, Flakoll PJ, Ikizler TA. Nutritional supplementation acutely increases albumin fractional synthetic rate in chronic hemodialysis patients. J Am Soc Nephrol. 2004;15:1920-6.

26. Masini ML, Bedogni G, Cecchetto G. Il processo assistenziale della malnutrizione in ospedale. Collana ANDID Ed. Universo. I Edizione 2011, Roma.

27. Manuale ANDID, International Dietetics and Nutrition Terminology. Un linguaggio condiviso per la pratica professionale. Italgraf s.r.l. I ${ }^{\mathrm{a}}$ edizione, Febbraio. 2014, Rubiera (RE). 